JOM, Vol. 69, No. 5, 2017

DOI: $10.1007 / \mathrm{s} 11837-017-2346-2$

(C) 2017 The Minerals, Metals \& Materials Society

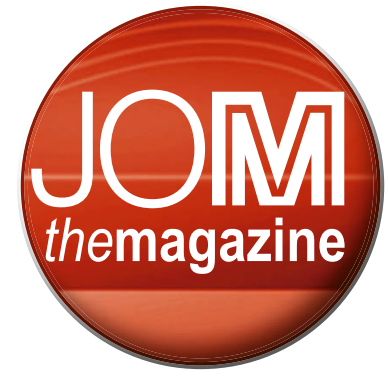

Shafiq Alam

Zafir Alam

Corby Anderson

David Andersson

Martin Anselm

Fatih Apaydin

Raymundo Arroyave

Ebrahim Asadi

Marcel Bachmann

David Bahr

Nagamani Jaya Balila

Parama Banerjee

Alan Barhorst

Stephen Bartolucci

Burak Basaran

Vishwas Bedekar

Ted Besmann

Sergey Betsofen

Yan Beygelzimer

Thomas Bieler

Nick Birbilis

Sudipta Biswas

Jake Blanchard

Wolfgang Blum

Kevin Bockenstedt

David Bourell

Patrick Bowen

\title{
Thanks to Our 2016 Peer Reviewers
}

JOM extends a sincere thank-you to the reviewers who helped to ensure that only the highest-quality articles were published in 2016. The professionalism and dedication of these volunteers is much appreciated.
Tom Broderick

Daniel Bufford

Daniel Builes

Carelyn Campbell

Fei Cao

Qing Cao

John Carsley

Jose Adilson Castro

Sbel Cetinel

Indrajit Charit

Eric Chason

Nikhilesh Chawla

Ailiang Chen

Biao Chen

Yang-yuan Chen

Youxing Chen

Atul Chokshi

Dianne Chong

Lokesh Choudhary

Young-Bum Chun

Peter Ciesielski

Amy Clarke

Kester Clarke

Peter Collins

Hervé Combeau

Brett Conner

Steve Constantinides
Steve Coryell

Eric Cotts

Dan Coughlin

Carl Cross

Santosh Dasika

Boyd Davis

Marc De Graef

Zhigan Deng

Karnika De Silva

Jhansi Rani Devireddy

Haoyan Diao

Susana Diaz

Shen Dillon

Saber DorMohammadi

Jaroslaw Drelich

David Dye

Venkata Kondaiah E

Mark Easton

Mohan Edirisinghe

Rod Eggert

John Elmer

Dinc Erdeniz

Mario Fafard

William Fahrenholtz

Adam Farrow

Saryu Fensin

Kip Findley

\section{Emre Firlar}

Stephen Foiles

Johan Foster

Miguel Fuentes-Cabrera

Yashuang Gao

Aimen Gheribi

John Gibbs

Gary Gladysz

Gultekin Goller

Sarma Gorti

Günter Gottstein

Christopher Gourlay

John Grandfield

Wanbing Guan

Xiaofei Guan

Lingling Guo

Manoj Gupta

Rajeev Gupta

Doga Gursoy

Bing Han

Mark Hardy

Sandip Harimkar

Md Hasnine

Eric Hintsala

Eric Homer

Soon-jik Hong

Norbert Hort 


\begin{tabular}{|c|c|c|c|}
\hline Mohammad Kamal & Daniel Kiener & Luhua Li & Dong Joon Min \\
\hline Hossain & Hojong Kim & Nan Li & Ken Mingard \\
\hline Yuri Hovanski & K. B. Kim & Zhibao Li & Satyendra Mishra \\
\hline Jeff Hoyt & Daniel King & Joseph Licavoli & Dave Mitchell \\
\hline John Hryn & Robert Klug & Yanli Lin & Surbhi Mittal \\
\hline C.S. Hsi & Benjamin Klusemann & Stephen Lindsay & Mohammad Ali \\
\hline Zhaorong Huang & Marko Knezevic & David Lipke & Mohtadi-Bonab \\
\hline Michael Hurley & Ozgur Kocaturk & Bin Liu & Takao Mori \\
\hline Robert Hyland & Markus Köhler & Fengchao Liu & Benjamin Morrow \\
\hline Dean llievski & Fatma Nese Kok & Rui Liu & William Mullins \\
\hline Eric Jägle & Katsuyoshi Kondoh & Yong Liu & Heather Murdoch \\
\hline Gaurav Jain & Hirokazu Konishi & Turab Lookman & Juan Nava \\
\hline Kailash Jajam & Hiren Kotadia & Dongping Lu & Neale Neelameggham \\
\hline Dongchan Jang & Milo Kral & Roger Lu & Ha-Duong Ngo \\
\hline $\begin{array}{l}\text { Daniel Salazar } \\
\text { Jaramillo }\end{array}$ & $\begin{array}{l}\text { Lorrie Krebs } \\
\text { Roman Kulagin }\end{array}$ & $\begin{array}{l}\text { Langli Luo } \\
\text { Shudong Luo }\end{array}$ & $\begin{array}{l}\text { Ronald Noebe } \\
\text { Michael Notis }\end{array}$ \\
\hline Vikram Jayaram & Alok Kumar & Dung Luong & $\begin{array}{l}\text { Khorgolkhuu } \\
\text { Odbadrakh }\end{array}$ \\
\hline Suwan Jayasinghe & Mehdi Lalpoor & Petra Maier & Stephanie O’Keeffe \\
\hline Thomas Jewett & Eric Lass & Dmitri Malakhov & Kanji Ono \\
\hline Baohua Jia & Olivier Lavigne & Abhinav Malasi & Harald Oosterhof \\
\hline Shian Jia & Byeong-Joo Lee & Vaso Manojlovic & Imre Norbert Orbulov \\
\hline Lin Jiang & Hyuck Mo Lee & Diego Mantovani & Takanari Ouchi \\
\hline Youfei Jiang & Jonghyun Lee & Steven Mates & Sidnei Paciornik \\
\hline Francis Johnson & Joonho Lee & Suveen Mathaudhu & Huilin Pan \\
\hline Utsavi Joshi & Myoung-Gyu Lee & Katter Matthias & Amit Pandey \\
\hline Vineet Joshi & Larry Lehman & Andrew Maximenko & B.D. Pandey \\
\hline Sathiskumar Jothi & Brad Lerch & James McGuffin-Cawley & Xin Pang \\
\hline Gillian Juleff & Amanda Levinson & Michael A. McGuire & Joohyun Park \\
\hline Surya Kalidindi & Bing Li & Brian McKay & Seong Jin Park \\
\hline Monica Kapoor & Changjun Li & Chamini Mendis & Somayeh Pasebani \\
\hline Ibrahim Karaman & Jian Li & Marco Messina & Pretesh Patel \\
\hline Mikko Kärkkäinen & Lan (Samantha) Li & Michael Miles & Sweetu Patel \\
\hline Michael Kassner & Ling Li & Janeček Milos & Soobhankar Pati \\
\hline
\end{tabular}




\begin{tabular}{|c|c|c|c|}
\hline Hong Peng & Flavio Ruffinatto & Niyanth Sridharan & Robert Wheeler \\
\hline Junjun Peng & Adrian Sabau & Sharada Srinivasan & Emma White \\
\hline Zhiwei Peng & Shima Sabbaghianrad & Aaron Stebner & Jesse White \\
\hline Koulis Pericleous & Ritesh Sachan & Mark Stoudt & Jim Wilcox \\
\hline Ray Peterson & Barry Sadler & Alton Tabereaux & Richard Williamson \\
\hline K. Pielichowski & Pierre Sallot & Mohammad Mahdi & Brian Wirth \\
\hline Evan Pineda & Peyman Samimi & Taheri & Thomas Wolfe \\
\hline Petrus Pistorius & Michael Sangid & Candan Tamerler & David Wong \\
\hline Wojciech Polkowski & Tomoko Sano & Fariborz Tavengarian & Albert T. Wu \\
\hline Gregory Poole & Soundarapandian & Michael Titus & Yang Xia \\
\hline Vinko Potocnik & Santhanakrishnan & Chandra Sekhar Tiwary & Xianghui Xiao \\
\hline Adam Powell & Graham Schaffer & Justin Tokash & Kelvin Xie \\
\hline Jeffrey Powers & Andrew Schissler & Vikas Tomar & Mingzhi Xu \\
\hline Chandra Prakash & Phil Schwarz & Jason Trelewicz & $\begin{array}{l}\text { Sagar Yadavall } \\
\text { Wen Yang }\end{array}$ \\
\hline A. K. Prasada Rao & Mohsen Seifi & lakovos Tzanakis & Xiao Yang \\
\hline Mohan Pujar & Alexander Senaputra & Kinga Unocic & Yifan Ye \\
\hline Saurabh Puri & Houfa Shen & Piyush Upadhyay & Huayi Yin \\
\hline Jonathan Puthoff & Nigel Shepherd & $\begin{array}{l}\text { Sreekumar Vadakke } \\
\text { Madam }\end{array}$ & Tokihiko Yokoshima \\
\hline Ma Qian & Fariba Sheyknjaberı & Maurits Van Camp & Qiulin Yu \\
\hline Tao Qu & Wenwu Shi & Joris Vandenhaute & Erfan Zalnezhad \\
\hline Dierk Raabe & Dongwon Shin & Mahesh Venkataraman & Sam Zelinka \\
\hline Bala Radhakrishnan & Meisha Shofner & Travis Voorhees & Steven Zeltmann \\
\hline Harish Radhakrishnan & $\begin{array}{l}\text { Devesh Dadhich } \\
\text { Shreeram }\end{array}$ & Hitesh Vora & $\begin{array}{l}\text { Li Zeng } \\
\text { Daojie Zhang }\end{array}$ \\
\hline Jeff Rao & Vasanth Chakravarthy & Samuel Wagstaff & Deliang Zhang \\
\hline Arne Petter Ratvik & Shunmugasamy & Lina Wang & Juhua Zhang \\
\hline Rajeev Rawat & Wim Sillekens & Mingyu Wang & Liying Zhang \\
\hline Raul Rebak & Gi-Dong Sim & Shengyu Wang & Mingming Zhang \\
\hline Thilo Rehren & Timothy Smith & Tao Wang & Yong Zhang \\
\hline Andrew Reid & Kiran Solanki & Yuxing Wang & Ji Zhao \\
\hline Kirk Rogers & Vikas Somani & Zhe Wang & Jianming Zheng \\
\hline Anthony Rollett & Suhas Somnath & Bryan Webler & Miaoyong Zhu \\
\hline Franz Roters & Benjamin Spencer & Xiaoliang Wei & Tie-Jun Zhu \\
\hline
\end{tabular}

